MILICA TAPAVIČKI-ILIĆ

Archäologisches Institut,

Belgrad, Serbien

mtapavic@sbb.rs

ANDREI GEORGESCU

Muzeul Naţional al Banatului,

Timişoara, Rumänien

andreigva@yahoo.com
903.25(282.243.7.043)"6387"

COBISS.SR-ID 264119308

Original research article

Received: April $13^{\text {th }} 2017$

Accepted: October $31^{\text {st }} 2017$

\title{
EIN BEISPIEL DER SPÄTEISENZEITLICHEN ORLEA- MAGLAVIT FIBEL AUS DER IMRE PONGRÁCZ SAMMLUNG
}

\section{ZUSAMMENFASSUNG}

Imre Pongrácz (1849-1903) war Major bei der Honvèd Infanetrie und Militärkommandant des Orșova Hafens. Während seines Lebens sammelte er über 6000 Antiquitäten, die überwiegend vom rechten Donau-Ufer, aus heutigem Serbien, stammen und von den sog. "Schatzjägern" gesammelt wurden. Immerhin fehlt für die meisten Objekte aus der Sammlung eine Fundplatzbestimmung. Ein bedeutender Bestandteil der Sammlung schließt unterschiedliche Fibeln mit ein, darunter auch etwa achtzig späteisenzeitlicher Fibeln. Die hier präsentierte Fibel gehört dem sog. "Orlea-Maglavit” Typ, der während der Späteisenzeit in Gebieten um die Donau auf dem Balkan vertreten war. Obwohl einige "Orlea-Maglavit" Fibeln schon veröffentlicht sind, werden im hiesigen Text einige Aspekte (Tragweise, Geschlechtsbestimmung der Fibelträger usw.) angesprochen, die bisher ungeklärt blieben.

\section{SCHLUSSWÖRTER: FIBEL, ORLEA, MAGLAVIT, SPÄTEISENZEIT, DONAU, SAMMLUNG.}

Imre Pongrácz oder Emerich von Pongrácz (1849-1903) war Major bei der Honvèd Infantrie und Militärkommandant des Orșova Hafens. Nachdem er in 1902. ohne Erfolg versuchte, seine Sammlung an das Banat Museum der Geschichte und Archäologie zu verkaufen, starb er plötzlich im folgenden Jahr. Nach langen und schweren Verhandlungen mit Pongráczs Erben gelang es dem Museum allmählich die Finanzmittel zu erzeugen, mit denen es über 6000 Antiquitäten kaufte, die einen Sammlungsbestandteil des gestorbenen Majors ausmachten. Die Mehrheit dieser Antiquitäten, die von Pongrácz gesammelt wurden, stammt vom rechten Donau-Ufer, aus heutigem Serbien. Dort hatte der Honvèd Offizier Verbindungen mit lokalen "Schatzjägern". Obwohl für die meisten Objekte aus der Sammlung eine Fundplatzbestimmung fehlt, erwähnt I. Pongrácz für einige die
Leute, die sie ihm verkauft haben und auch den Fundort aus dem die Objekte stammen. Einige von den Späteisenzeitlichen Funde aus der "Pongrácz Sammlung" wurden früher kurz erwähnt und in dem Sinne veröffentlicht.

Schon im 19. Jahrhundert wurden alle Funde aus der Imre Pongrácz Sammlung gezeichnet, darunter auch die Fibel, die hier weiter präsentiert wird (Abb. 1). Sie trägt die Inventarnummer 1729 (Abb. 2) und wurde aus Bronze hergestellt. Ihre Dimensionen sind: L: $5,2 \mathrm{~cm}, \mathrm{~B}: 1,0 \mathrm{~cm}$, Bügelbreite (maximal): $2,0 \mathrm{~cm}$.

Der Fuß und die Nadel fehlen, sowie eine Hälfte der Spiralwindung.

Dieses Stück gehört zu dem sog. Orlea-Maglavit Fibeltyp. Die Hauptmerkmale dieses Typs sind ein Kopf mit Spiralwindung (üblicherweise sechs Spiralen), ein langer, oval oder rhombisch verbrei- 


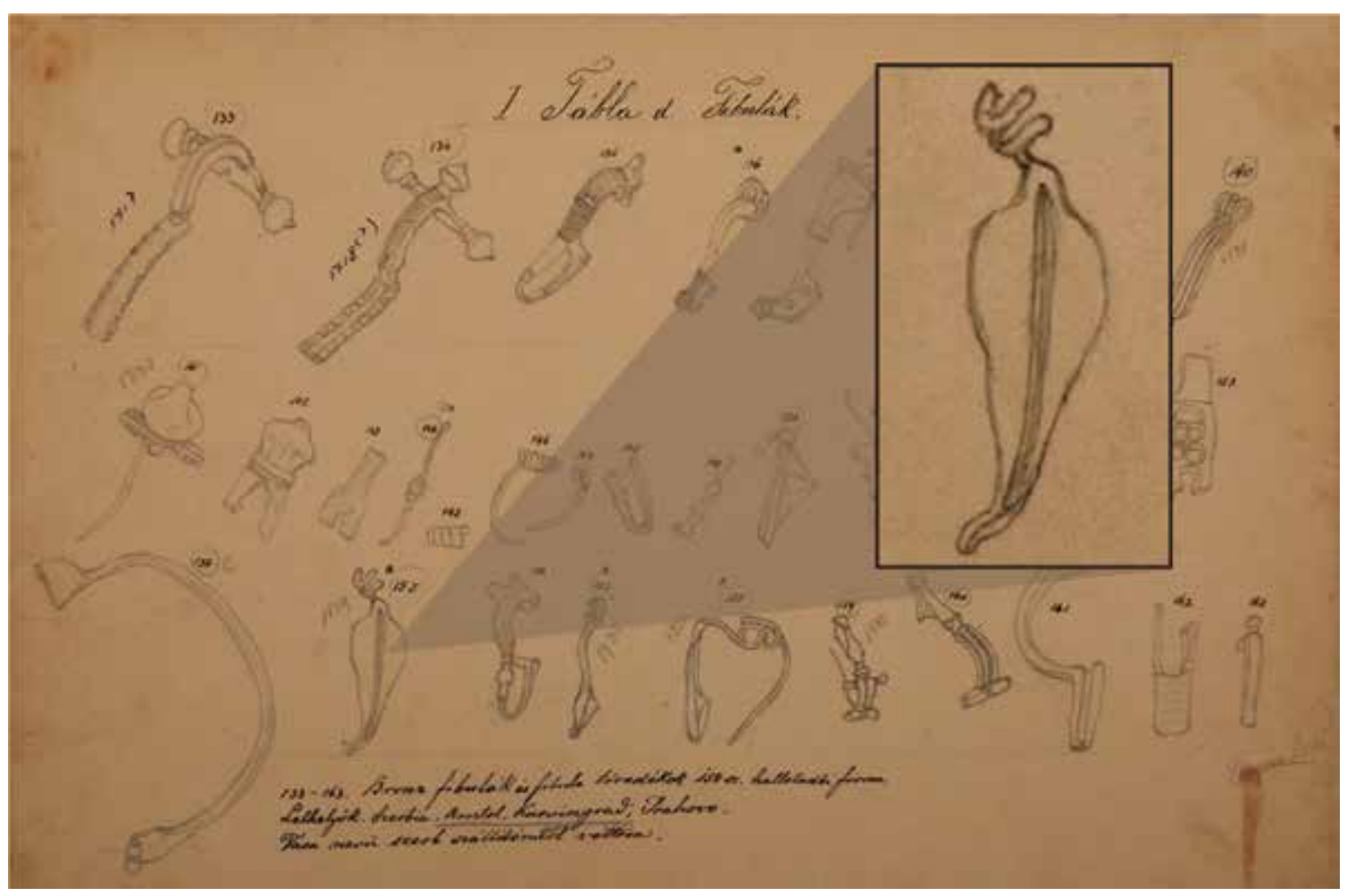

Abb. 1 - Zeichnung der Fibel Inv. Nr. 1729 aus der Imre Pongrácz Sammlung (Archiv des Muzeul Naţional al Banatului, Timişoara).

teter Bügel mit einer länglichen Rille, zurückgezogener Fuß mit einer kugel- oder bikonischen Verdickung und eine längliche und verdünnte Endung, die fast bis zum Bügelknie hinreicht. Manchmal tragen solche Fibeln eine eingeritzte Verzierung von kleinen Dimensionen. Sie wurden aus Bronze hergestellt und werden in Spätlatène C/Frühlatène D Zeit datiert, bzw. die erste Hälfte des 1. Jh. v. Chr. (Rustoiu 1997, 38, Popović 1992, 321).

Die Orlea-Maglavit Fibeln treten häufig auf dem Territorium der Padea-Panaghiurski Kolinii Kulturgruppe auf, das als ihr Entstehungsgebiet gilt (Rustoiu 1997, 38). Die meisten Stücke wurden im Donaugebiet ausgegraben, im Süden Olteniens, Nord und Nordwesten Bulgariens und am Eisernen Tor. In anderen Worten sind die Fibeln von diesem Typ für die östlichen Grenzgebiete der keltischen Welt typisch, bzw. südwestliches Rumänien, nordwestliches Bulgarien und Ostserbien. Sie werden als "keltisch" bezeichnet, obwohl sie manchmal schwer mit ihren Parallelen im keltischen Kerngebiet in Zusammenhang zu bringen sind. Vor allem ist dies wegen großen chronologischen Unterschieden. Die Bevölkerung, die in den Grenzgebieten siedelte, war wegen Ferne, aus politischen oder anderen Gründen häufig nicht in der Lage, die letzte Mode und Trends, sowie technologische Neuigkeiten des Kerngebietes zu folgen. Außerdem kam es zu Konservativismus, überwiegend bei den Adeligen, die die Herstellung einiger schon veralteten Typen für eine längere Zeit beibehielten. Bestimmte, aus keltischen Beispielen stammende Fibeltypen, besaßen eine eigene, lokale Entwicklung und sollten eigentlich als unabhängig betrachtet werden.

Dies geschah ebenso mit den Fibeln vom Typ Orlea-Maglavit, die an das Ende des 2. und in die erste Hälfte des 1. Jh. v. Chr. zu datieren sind (Popović 1992, 322). Ihr Ursprung ist bei den Frühlatènefibeln mit rhombischem Bügel zu suchen und deswegen besaß dieser Typ schon bei seiner Entstehung eine archaische Form. Eine ähnliche chronologische Bestimmung ist auch bei Rustiou zu finden (Rustoiu 1997, 38), der weiter- 


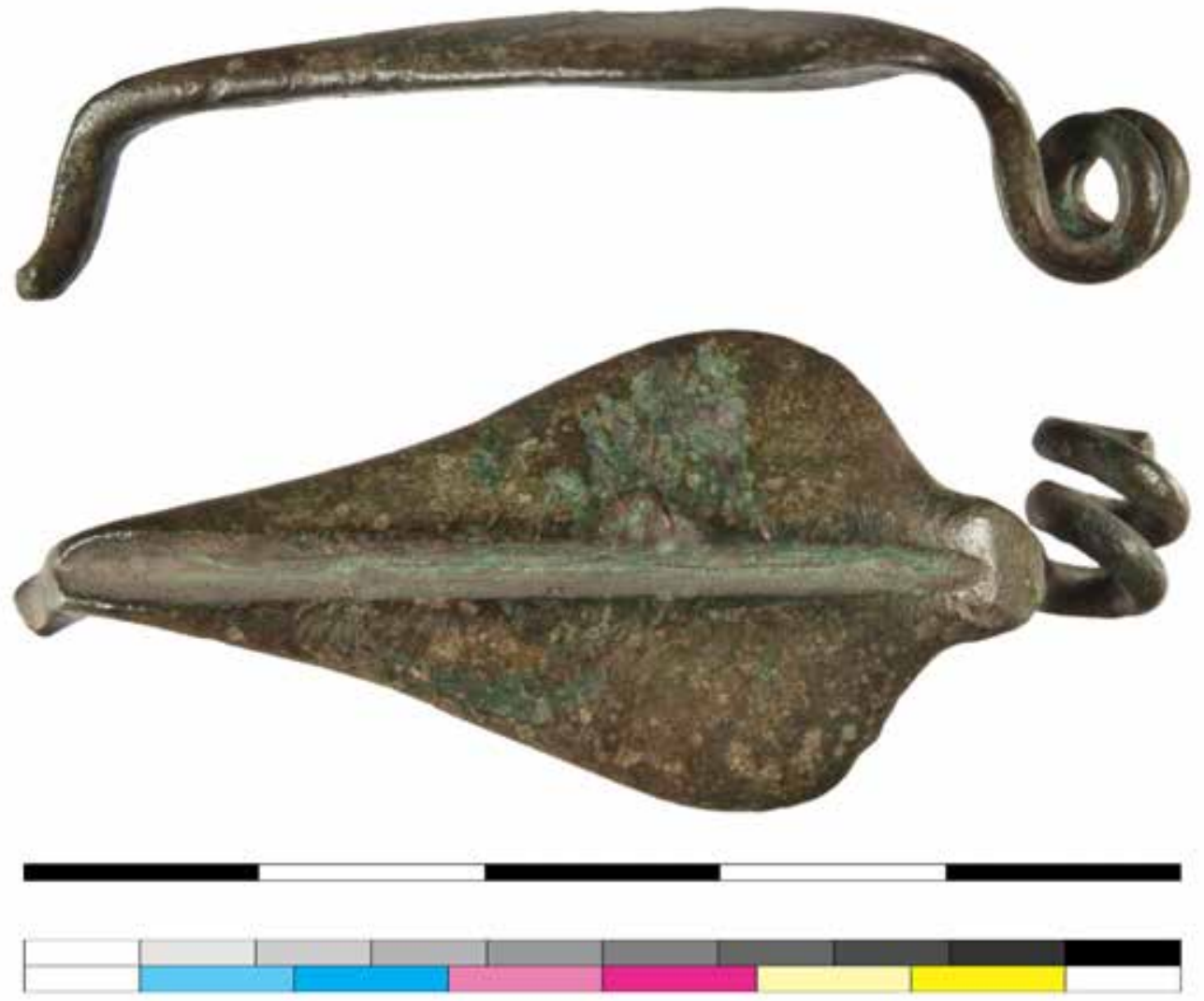

Abb. 2 - Fibel Inv. Nr. 1729 aus der Imre Pongrácz Sammlung (Foto V. Ilić).

hin seine Bestimmung auf denjenigen von Zirra (Zirra 1971, 544) basiert.

Die Rolle der Orlea-Maglavit Fibeln wurde von Rustoiu diskutiert. Er betrachtet sie als skordiskisches Einfluss auf die Daker, das im 2. Jh. v. Chr. vom Gebiet der kleinen Skordiskern in Richtung Osten und Südosten verlief. Solche Einflüsse sind angeblich mit militärischen Bewegungen in Zusammenhang zu bringen, während dessen auch Moesi und Triballi beeinflusst wurden. Geografisch gesehen, umfassten die Einflüsse das nordund nordwestliche Bulgarien, das Donaugebiet am Eisernen Tor, Oltenien, westliches und südliches Muntenien und das südwestliche Transsylvanien (Rustoiu 1997, 78) (Karte).

Obwohl die Fibeln aus der Pongracz Sammlung ausnahmslos Einzelfunde ohne archäologischen Kontext darstellen, ist es bekannt, dass sie aus der Umgebung oder direkt aus Orşova stammen. Die hier besprochene Fibel vom Typ Orlea-Maglavit Fibel passt, geografisch gesehen, ausgezeichnet in den oben genannten Rahmen des Donaugebietes am Eisernen Tor.

Analogien wurden in Serbien (Kostolac-Pećine, Mala Vrbica-Ajmana), Bulgarien (Carevec, Ivanski-Zlokučen, Malamir) und auch Rumänien (Govora-Sat, Saltia, Maglavit, Orlea, Cetace, Lişteava) gefunden (Popović 1992, 323-324).

Die Tragweise der Orlea-Maglavit Fibeln ist völlig schwer zu erschließen. In diesem geografischen Raum und im Gegenteil zu Römerzeit fehlen diesbezügliche bildliche Darstellungen. $\mathrm{Da}$ die Orlea-Maglavit Fibeln immer als Einzelstücke zutage kamen, kann ausgeschlossen werden, dass sie paarweise getragen wurden. Trotzdem ist nicht klar, ob sie als Verschluss- oder Zierspange gedient haben. Bei einem hochgewölbten Bügel besteht zwischen Bügel und Nadel ein genügend 
großer Raum, um zwei Lagen selbst eines dicken Stoffes einzuschließen (Riha 1979, 41). Daher könnten Typen mit hochgewölbtem Bügel als "Mantelspangen" bezeichnet werden. Ebenso entsteht genügend Raum bei einem geknickten oder bei einem langen, gestreckten Bügel, wie gerade der Fall mit der Orlea-Maglavit Fibel aus der Pongracz Sammlung ist.

Neben Tracht - und Schmuckfunktion konnte den Fibeln auch eine magische Bedeutung zukommen, so Stücken mit apotropäischen Zeichen («Augen», Tierköpfe, Mondsymbole) (Riha 1979, 42). Mit diesem Gedanken können auch die Fibeln vom Orlea-Maglavit Typ in Zusammenhang gebracht, da einige von denen, wie z. B. das Stück aus Mala Vrbica - Konopište, mit "Augen" geschmückt sind (Popović 1992, 325, Taf. 1/12). Selbst die Rille mitten auf Bügeln dieser Fibeln könnte als Mondsymbol gedeutet werden.

In Anlehnung an diese Tragweise wird auch hier, genauso wie für die anderen Fibeltypen angenommen, dass der Fibelkopf nach unten, der Fuß nach oben gerichtet war. Es könnte aber sein, dass bestimmte Typen, sogar auch der hier besprochene, auch anders gerichtet, z. B. quergestellt, getragen wurden.

Weiterhin blieb es immer noch unbekannt, ob sie zu Frauen- oder Männertracht gehörten. Die meisten Funde der Orlea-Maglavit Fibeln gehören zu Einzelfunden, wie diejenigen aus Orlea, Maglavit, Salcia, Lişteava und Scorniceşti (Măndescu 2004, 13). Als Grabbeigaben, wie etwa aus der Nekropole Mala Vrbica - Ajmana (Popović 1992, 323), kommen sie immer in Gräbern mit geschlechtunspezifischen Funden vor, wie Tongefäßen oder Messern.

Neben der Tatsache, dass die Fibeln der La Tène Scheme üblicherweise für eine Datierung und Chronologie anderer Funde genutzt werden, werden sie auch als eine Art ethnischer Indikatoren bezeichnet. Die Fibeln der Spätlatèneschema werden generell den Kelten aus Mitteleuropa zugeschrieben, die unter anderem ebenso nach Osten umsiedelten (Zirra 2011, 4). In einem solchen
Falle und als ein isoliertes Kriterium, können die Orela-Maglavit Fibeln keinen nötigen Beweis anbieten, um ausschließlich und ohne weiteres den Ostkelten, bzw. den Skordiskern, zugeschrieben zu werden. Sie können eher als eine lokale Entwicklung, die in einer ethnisch gemischten Umgebung entstand, bezeichnet werden.

\section{SUMMARY \\ AN EXAMPLE OF LATE IRON AGE BROOCH OF THE ORLEA- MAGLAVIT TYPE FROM THE IMRE PONGRÁCZ COLLECTION}

Imre Pongrácz (1849-1903) was a major of the Honvèd Infantry and the commander of the Orșova harbor. During his lifetime and his service as an officer, he collected over 6000 antiquities, mostly coming from the right Danube bank, actually from modern Serbia. All of the finds were collected by the so-called "treasure-hunters" and this is why most of the finds lack precise data regarding places of their origin.

An important part of the Imre Pongrácz collection, kept at the Muzeul Naţional al Banatului in Timişoara (Romania) consists of various fibulas. Among them, some eighty fibulas belong to the Late Iron Age. The fibula presented in this paper belongs to the so-called "Orlea-Maglavit" type, that was spread during this period along the Danube valley in the Balkans. Several "Orlea-Maglavit" fibulas were already published and their dating and ethnic belonging were discussed. However, in this paper, several other aspects have been discussed, like the way in which they were worn or the gender of people who wore them. These and some other aspects remained unclarified and unknown.

$$
* * *
$$

Arheologija i prirodne nauke (Archaeology and Science) is an Open Access Journal. All articles can be downloaded free of charge and used in accordance with the licence Creative Commons 


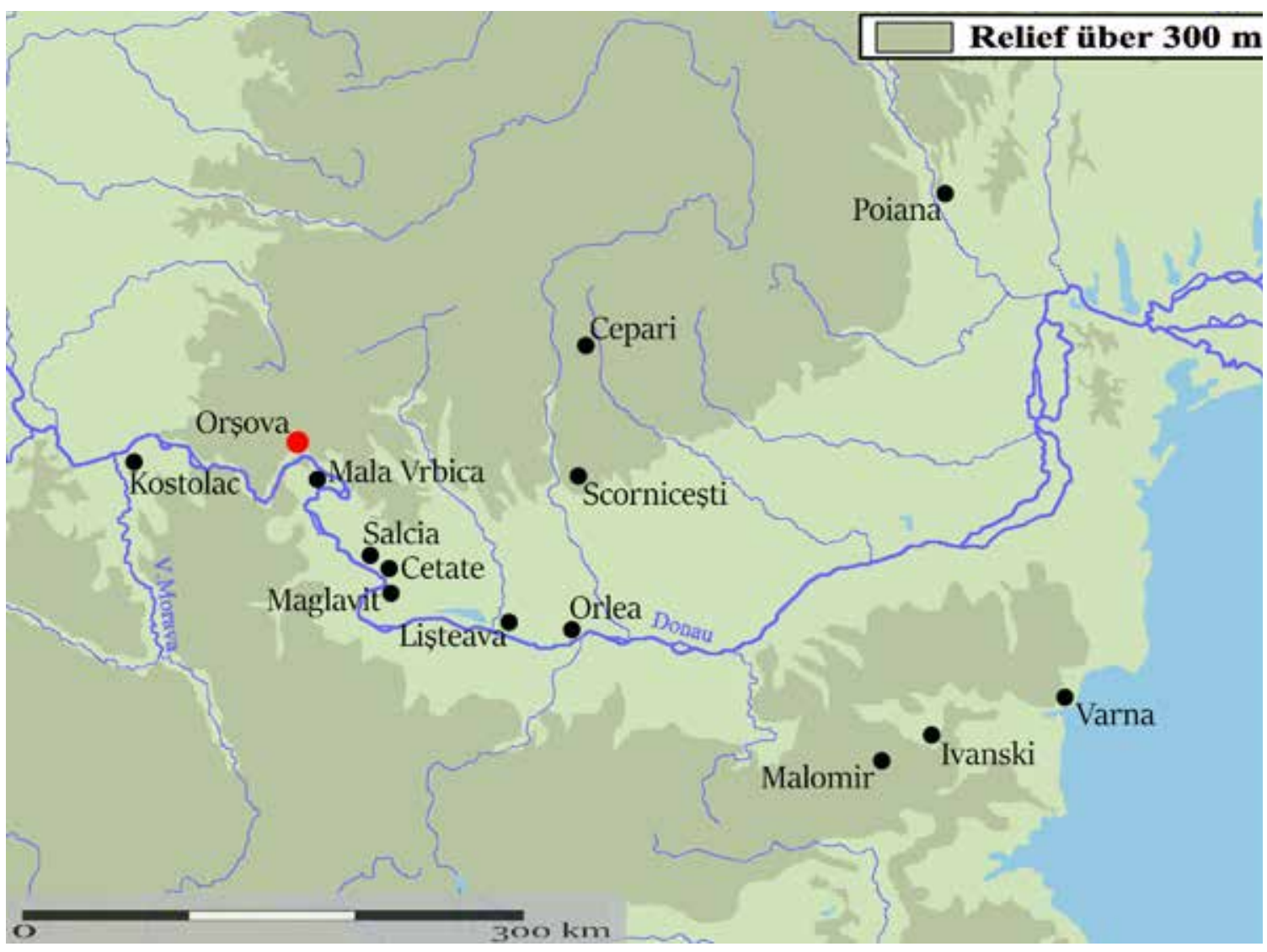

Karte - Fundorte der “Orlea-Maglavit” Fibeln (Karte teilweise nach Rustoiu 1997, S. 179 und Măndescu 2004, Fig. 2).

- Attribution-NonCommercial-NoDerivs 3.0

Serbia (https://creativecommons.org/licenses/bync-nd/3.0/rs/.

Časopis Arheologija i prirodne nauke je dostupan u režimu otvorenog pristupa. Članci objavljeni $\mathrm{u}$ časopisu mogu se besplatno preuzeti sa sajta i koristiti u skladu sa licencom Creative Commons - Autorstvo-Nekomercijalno-Bez prerada 3.0 Srbija (https://creativecommons.org/licenses/bync-nd/3.0/rs/.

\section{BIBLIOGRAPHIE}

\section{Măndescu, D. 2004}

Căteva consideraţii relative la tipul de fibule Orlea-Maglavit, Danubius XXII: 14.

\section{Popović, P. 1992}

Fibule tipa "Orlea-Maglavit", Zbornik Narodnog Muzeja XIV/1, Archäologie: 320, T. I, 7.
Riha, E. 1979

Die römischen Fibeln aus Augst und Kaiseraugst, Forschungen in Augst 3, Augst.

\section{Rustoiu, A. 1997}

Fibulele din Dacia preromană (sec. II î.e.n. - I e.n.), Bibl. Thrac. 22, Bucureşti.

\section{Zirra, V. 1971}

Latenezeit in Rumänien, Arheologicky Rozhledy 23: 529-547.

\section{Zirra, V. 2011}

Research Stage of the Le Tène and Thracian Scheme Fibulae in Romania - Dynamics and exclusion. Critical general Approach, WSEAS Transactions on Environment and Development, Issue 1, Volume 7, http://www.wseas.us/e-library/transactions/ environment/2011/52-365.pdf, accessed February 2017. 


\section{REZIME}

\section{PRIMERAK KASNOLATENSKE} FIBULE TIPA ORLEA-MAGLAVIT IZ ZBIRKE "IMRE PONGRAČ"

Imre Pongrač (1849-1903) je bio pešadijski Honved oficir i vojni komandant luke u Oršavi. Tokom života, a pre svega tokom oficirske službe, sakupio je preko 6000 predmeta koji većinom potiču sa desne obale Dunava, iz današnje Srbije. Sve te predmete su pronašli tzv. "lovci na blago", pa zbog toga za većinu nalaza iz ove zbirke nedostaje precizno definisano mesto nalaza.

Značajan deo ove zbirke se čuva u Narodnom muzeju Banata u Temišvaru (Muzeul Naţional al Banatului - Timişoara), a sastoji se od velikog broja različitih fibula. Među njima je oko osamdeset fibula iz kasnog gvozdenog doba. Fibula koja je ovde predstavljena pripada tzv. tipu "Orlea-Maglavit”, koji je tokom kasnog gvozdenog doba bio rasprostranjen u oblasti balkanskog Podunavlja. Nekoliko fibula tipa "Orlea-Maglavit" je već publikovano, tako da je određeno njihovo datovanje i postojali su pokušaji određivanja njihove etničke pripadnosti, kao i način na koji su nošene i pol osoba koje su ih nosile. Ipak, mnogi od ovih faktora ostali su nerazjašnjeni i nepoznati. 